\title{
Characterisation of pain in people with hereditary neuropathy with liability to pressure palsy
}

Darren Beales $^{1} *$ (Orcid: 0000-0002-7176-4644)

Robyn Fary ${ }^{1}$

Cameron Little ${ }^{1}$

Shruti Nambiar ${ }^{1}$

Hakon Sveinall ${ }^{1}$

Yen Leng Yee ${ }^{1}$

Brigitte Tampin $^{1,2,3}$

Tim Mitchell ${ }^{1}$

${ }^{1}$ School of Physiotherapy and Exercise Science, Curtin University. GPO Box U1987, Perth, Western Australia, Australia 6845

${ }^{2}$ Physiotherapy Department, Sir Charles Gairdner Hospital, Hospital Ave, Nedlands, Western Australia, 6009, Australia

${ }^{3}$ Faculty of Business Management and Social Sciences, Hochschule Osnabrück, University of Applied Sciences, Caprivistr. 30A, 49076 Osnabrück, Germany

* Corresponding Author

Dr Darren Beales. School of Physiotherapy and Exercise Science, Curtin University. GPO

Box U1987, Perth, Western Australia 6845.

Tel.: +61 892664644

Fax: +61 892663699

E-mail: D.Beales@curtin.edu.au 


\begin{abstract}
Hereditary neuropathy with liability to pressure palsy (HNPP) has historically been considered a pain free condition, though some people with HNPP also complain of pain. This study characterised persistent pain in people with HNPP. Participants provided crosssectional demographic data, information on the presence of neurological and persistent pain symptoms, and the degree to which these interfered with daily life. The painDETECT and Central Sensitization Inventory questionnaires were used to indicate potential neuropathic, central sensitisation and musculoskeletal (nociceptive) pain mechanisms. Additionally, participants were asked if they thought that pain was related to/part of HNPP. 32/43 (74\%) subjects with HNPP had persistent pain and experiencing this pain in the last week. Of those with pain, 24 (75\%) were likely to have neuropathic pain and 27 (84\%) were likely to have central sensitisation. All 32 participants felt that their pain could be related to/part of their HNPP. Significant negative impact of the pain was common. Pain characterisation identified neuropathic pain and/or central sensitisation as common, potential underlying processes. Pain may plausibly be directly related to the underlying pathophysiology of HNPP. Further consideration of including pain as a primary symptom of HNPP is warranted.
\end{abstract}

\title{
Key Words
}

hereditary neuropathy with liability to pressure palsy; pain; neuropathic; central sensitisation 


\section{Introduction}

Hereditary neuropathy with liability to pressure palsy (HNPP) is an autosomal dominant disorder affecting peripheral nerves $[5,9,10]$. Definitive diagnosis is made via genetic testing. Typically symptoms include recurrent sensory loss and/or focal weakness related to the affected peripheral nerve, manifesting clinically with muscle atrophy, decreased tendon reflexes, and in some cases secondary orthopaedic deformities (such as pes cavus) $[1,5,9$, 10]. Polyneuropathy presentations are thought to be less common [13]. Symptoms are described as transient, usually lasting several hours to months, though incomplete recovery is reasonably common [1]. It has been suggested the overall impact of the disorder on quality of life is low [21].

Hereditary neuropathy with liability to pressure palsy is generally considered a pain-free disorder $[1,4,10,18]$. However, a few studies have suggested pain may be an issue for people with HNPP. Case-study evaluations of the onset of HNPP describe pain as a potential primary symptom at initial presentation $[6,26,31]$. In a more detailed retrospective review of medical records in 39 people with genetic confirmation of HNPP, six (15\%) reported that pain was their primary initial complaint, with three others reporting pain at a later time [9]. The pain in these nine individuals was deemed neuropathic, seemingly based on clinical presentation. Another retrospective review of 32 people with genetic confirmation of HNPP provided a more detailed characterisation of pain [30]. Twenty four (75\%) people reported pain. Based on pain descriptors, nine (28\%) presented with musculoskeletal pain only, 10 (31\%) with neuropathic pain only and five (16\%) with both musculoskeletal and neuropathic pain [30]. Further, nine people (28\%) met the 1990 American College of Rheumatology criteria for fibromyalgia syndrome. 
As pain may be a significant symptom for some people with HNPP, mechanistic links between HNPP and pain might be important. There appear to be four potential reasons for pain in HNPP. Firstly, given HNPP is by definition a neuropathy, it is plausible that neuropathic pain mechanisms could underpin any potential relationship between HNPP and pain $[9,30]$. Alternately or concurrently, altered central processing (central sensitisation) could potentially be related to underlying neuropathic process in HNPP, contributing to pain in HNPP [30]. Thirdly, local tissue stress caused by prolonged weakness or secondary joint deformity might facilitate peripheral nociception consistent with musculoskeletal pain [30]. Lastly, comorbidity of HNPP and pain could be co-incidental, without any shared mechanistic links.

The primary purpose of this study was to provide a more detailed characterisation of pain in people with HNPP to compliment emerging literature in this area. Specific aims were; (1) to determine the prevalence of participants with HNPP who experience persistent pain and pain in the last week, (2) characterise pain in these participants with persistent pain and pain in the last week, in relationship to potential pain mechanisms, and (3) assess for any correlation between the levels of neurological and pain interference in these same participants. Improved understanding of pain in HNPP would be beneficial to healthcare practitioners and people with HNPP alike.

\section{Methods}

\section{Study design and participants}

For this cross-sectional study, participants were recruited through convenience sampling, using closed social media groups on Facebook (HNPP Help; HNPP Australia). Individuals were 
included if they reported a diagnosis of HNPP confirmed via genetic testing, were 18 years and older, and were able to read and write English. Individuals were excluded if they had other causes of neuropathy (for example: alcoholism, malnutrition, Vitamin B12 deficiency, and systematic diseases such as diabetes and other endocrinopathies), or other neurological diseases (for example: Charcot Marie Tooth Type 1A). All participants provided informed consent. Ethical approval was granted by the Curtin University Human Research Ethics Committee (Approval\# HRE2016-0118).

Participants were recruited via an invitation posted to the closed Facebook groups. The premise of the study was to characterise symptoms associated with HNPP without any specific emphasis on pain, to avoid preferential engagement of people with HNPP who do experience pain. Potential participants were directed to an online questionnaire hosted on Qualtrics software. Data collection occurred between $18^{\text {th }}$ July 2016 to $1^{\text {st }}$ August 2016.

\section{Variables}

Participant characteristics data included age; sex; time since diagnosis; employment status; and if the person was not working, whether HNPP was the reason for not working. Additionally, the types of neurological symptoms experienced (tingling, pins and needles, numbness, twitching, weakness) and self-reported health practitioner diagnosed comorbidities were recorded.

\section{Pain}

Participants were asked if they had ever experienced bodily pain. Only those participants who reported experiencing pain were then asked further questions relating to the characterisation of pain. Information sought included identification of the areas of the body 
affected by pain from a list. To determine the presence of persistent pain [24], participants were asked; (1) "Have you ever experienced persistent pain? (Persistent pain is defined as experiencing pain on most days in the last three months)", and (2) "Have you experienced pain in the last week". Participants had to answer affirmatively to both of these questions to be considered as having persistent pain and pain in the last week. This definition was used to include participants with greater likelihood of ongoing difficulties related to pain, and exclude those with more trivial or transient painful episodes [24].

The Brief Pain Inventory Pain (BPI) $[8,28]$ is widely used to assess pain, and is not condition specific. The BPI-Pain Severity Scale (BPI-PSS) comprises four questions, asking about worst pain, last pain, least pain and current pain. Each construct is scored from 1-10 (from 'no pain' to 'worst possible pain'). The BPI-Pain Interference Scale (BPI-PIS) was used to assess the effect of pain on function. The BPI-PIS comprises seven questions, each scored from 1-10 (from 'does not interfere' to 'strongly interferes'). Dimensions include interference with general activity, mood, walking ability, normal work, relations with other people, sleep and enjoyment of life. The BPI-PSS and BPI-PIS were reported as the average of the four and seven questions respectively [8]. The BPI has established validity and reliability $[8,28]$.

The painDETECT questionnaire (PD-Q) was used to determine the potential presence of neuropathic pain [11]. The PD-Q consists of nine questions with an overall score ranging from $0-38$. A score of $0-12$ suggests a neuropathic component to the presentation is unlikely ( $<15 \%$ likelihood) and the pain is more likely to be nociceptive, 13-19 being unclear, and 1938 indicating a neuropathic component to the presentation is likely (>90\% likelihood) [11]. Originally developed for patients with lower back pain, PD-Q is validated to predict the 
presence of neuropathic pain in the chronic pain population (sensitivity $84 \%$, specificity $84 \%$ )

[11]. The reliability of the English version of PD-Q has been established [27].

The Central Sensitization Inventory (CSI) was used to assess potential altered central processing (central sensitisation) [16, 19, 22]. The CSI consists of 25 questions, rated on a five-point Likert scale ('never', 'rarely', 'sometimes', 'often', 'always'). A cut off score of 40/100 has been shown to best distinguish between a central sensitization group and a comparison sample in a study of 121 participants from a pain clinic and 129 pain free participants from a university [19] with area under the curve $(A \cup C)=0.86$, sensitivity $81 \%$, specificity $75 \%$. As such, participants with a score less than 40 were classified as being unlikely to have central sensitisation and 40 or above as likely to have central sensitisation. A separate section of the CSI collecting data on doctor diagnosed comorbidities was not used for this paper.

Additionally, participants with pain were asked "If you experience pain, do you think your pain is related to or part of HNPP?" ('always', 'sometimes', 'never'). They were also asked “Have you seen/spoken to a healthcare practitioner about your pain, or sought help/treatment for your pain?" ('yes', 'no').

\section{Neurological symptoms}

Participants selected the areas of their body affected by neurological symptoms from a list. Review of the literature did not locate a questionnaire suitable for online distribution in this cohort to assess the interference of neurological symptoms on daily function. Consequently, we elected to establish the interference caused by neurological symptoms on function by adapting the BPI-PIS from pain symptoms to neurological symptoms. The question "Select 
the one number that describes how, during the past week, pain has interfered with each of the following" from the BPI-PIS, was modified to "Select the one number that describes how, during the past week, neurological symptoms have interfered with each of the following". We utilized the same seven questions, responses, and the same mean scoring calculation as the BPI-PIS.

\section{Analysis}

Analysis was performed in STATA 14.2 for Mac. Participant characteristics were reported using descriptive statistics for the entire cohort who met the inclusion criteria. Descriptive statistics were also used for the first aim, to report the prevalence of persistent pain and pain in the last week. Cross tabulation was then used to define the pain group of interest, being those who had both persistent pain and pain in the last week. Further analysis was only performed on this group.

For the second aim, relating to only those participants reporting persistent pain in the last week, mean scores were calculated for the BPI-PSS, BPI-PIS and neurological symptom interference. Participant categorisation from both the PD-Q and CSI, and if they thought pain was related to/part of HNPP, were reported descriptively. For the third aim, Pearson's correlations were used to assess potential associations between pain interference and neurological symptom interference for the overall scores and each of the seven individual interference dimensions. Strength of correlations were interpreted in accordance with recognised guidelines [3], with an alpha value of 0.05 . 


\section{RESULTS}

Figure 1 indicates the flow of participants through the study. A total of 43 met the inclusion criteria and completed the full questionnaire. Participant characteristics of this cohort are presented in Table 1.

Two participants $(5 \%)$ reported they had not experienced bodily pain. Nine participants (21\%) reported pain, but this pain was neither persistent or present at the time of completing the questionnaire. Thirty two $(74 \%)$ met the criteria of having persistent pain and experiencing this pain in the last week. The participant characteristics of this subset are also presented in Table 1. Further results relate to these 32 participants.

Areas of neurological symptoms and pain are indicated in Table 2. Neurological symptoms were reported more frequently in the extremities. Pain was also experienced frequently in the extremities, but was reported more than neurological symptoms in the spine and shoulders also.

Table 3 provides information on the characterisation of pain, as well as scores for the BPIPSS, BPI-PIS and neurological symptom interference. Twenty four (75\%) participants were likely to have neuropathic pain based on categorisation from the PD-Q. Twenty seven (84\%) participants were likely to have central sensitisation, based on categorisation from the CSI. All 32 participants felt that their pain could be related to/part of their HNPP and $28(88 \%)$ had sought health care for this pain. 
Pain interference and neurological interference were commonly reported together by participants. Significant correlations between the BPI-PIS and neurological interference were found for the total scores and all the individual interference dimensions (Table 4). The correlation for the total scores was high [3]. For the individual dimensions, high correlations were found for mood, relations with other people, sleep and enjoyment of life [3]. Moderate correlations were found for walking ability and normal work [3]. The correlation for general activity was low [3].

\section{DISCUSSION}

Generally, HNPP is considered to be a pain free neuropathy $[1,4,10,18]$. This study identified a cohort of people with HNPP, of which $74 \%$ reported having persistent pain and had experienced pain in the week preceding taking part in this study. This is consistent with previous finding following a review of medical records in a similar sized cohort [30], though it is not clear that those participants were complaining of persistent pain. Our finding suggests pain may be more common in individuals with HNPP than previously thought, and is more common than seen in the general population [24]. A key question is could the pain experienced be part of the HNPP presentation, rather than a co-incidental comorbidity?

\section{Potential relationships between HNPP and pain}

The pain was characterised as likely neuropathic for $75 \%$ of people in this study using the PD-Q. This aligns to the prior suggestion that pain in people with HNPP could be neuropathic in nature based on pain descriptors [30] or clinical presentation [9]. By definition, neuropathic pain results from a lesion or disease of the somatosensory nervous system [12]. 
Changes in myelin have been identified as part of the pathological process in HNPP [23], and have also been identified as a contributor to neuropathic pain mechanisms [29]. This provides a plausible explanation for a direct link between the pathology of HNPP and pain.

Based on categorisation from the CSI, 27 (84\%) of participants were likely to have central sensitisation. It is important to note that central sensitisation and neuropathic pain are separate pain processes, though each may result in similar symptoms [20]. Central sensitisation has also been proposed as a link between HNPP and pain [30]. Altered peripheral processes in neuropathy have the potential to contribute to more nervous system wide changes and sensitisation [25], which are characteristics of central sensitisation. This relationship could explain the finding of the likely presence of central sensitisation in a large proportion of individuals with HNPP with persistent pain.

Yilmaz et al (2015) contended that comorbidity of HNPP and fibromyalgia may be mediated by neuropathic pain. Fibromyalgia is a widespread pain disorder thought to have altered central pain processing (central sensitisation) as a potential underlying mechanism in pain in fibromyalgia $[2,7]$. In that sample, $28 \%$ of people with HNPP met the criteria for fibromyalgia. In our study, only $12 \%$ of participants reported a health professional diagnosis of fibromyalgia, but a larger number could have potentially met the diagnostic criteria for this disorder.

Neuropathic pain and central sensitisation were the most prominent findings from the PD-Q and CSI respectively. However, this does not rule out the potential for peripheral 
nociceptive/local musculoskeletal pain secondary to local tissue stress caused by prolonged weakness or secondary joint deformity. Co-existence of nociceptive and neuropathic pain processes has been recognised across numerous forms of painful peripheral neuropathies [15]. Somatosensory profiling using quantitative sensory testing may aid in the differentiation of nociceptive and neuropathic pain components [14].

\section{Impact of pain in those with HNPP}

The high prevalence of persistent pain with this pain experienced in the prior week, and the characterisation of the pain indicating high likelihood of neuropathic pain and/or central sensitisation, suggests that in this group of people with HNPP pain is unlikely to be a coincidental comorbidity. Further, all 32 participants with persistent pain considered the pain could be related to or part of HNPP (Table 3). This highlights a potential mismatch between the beliefs of individuals with HNPP about pain as part of their disorder, and the traditional description of HNPP as a pain free disorder. With $28(88 \%)$ of participants having sought medical assistance for pain, there is also potential for mismatch in beliefs between the individual with HNPP and the health care practitioner. In addition to understanding that pain might be related to HNPP, the health care practitioner may need to be skilled at identification of different pain characteristics (nociceptive, neuropathic, central sensitisation) to facilitate effect management strategies [20].

The mean score for the BPI-PSS was 4.9 (standard deviation 1.9), and for the BPI-PIS was 5.5 (standard deviation 2.5). This is comparable to results from a study using a version of the BPI for painful diabetic peripheral neuropathy. In that cohort of 255 people with diabetic peripheral neuropathy, the BPI-PSS average was 4.7 (standard deviation 2.6), and the BPI-PIS 
average was 4.9 (standard deviation 2.8) [32]. These authors used the BPI-PSS worst pain question to categorise the pain as mild (0-3), moderate (4-6) and severe (7+). While it was not the purpose of this study to recreate this categorisation, with post-hoc analysis of the data in this study (via the worst pain question in the BPI) eight participants with HNPP would have moderate symptoms and 23 would be categorised with severe symptoms. This suggests pain can be a significant problem for some people with HNPP.

In a similar manner, the burden of neuropathic pain has been described with use of the BPI. In 602 participants with a broad array of diagnoses resulting in clinical determination of neuropathic pain, the BPI-PSS average was 4.8 (standard deviation 2.1), and the BPI-PIS average was 4.5 (standard deviation 2.4) [17]. Higher severity was associated with poorer health related quality of life, higher levels of disrupted employment and more frequent physician visits [17]. Given similar pain severity and inference scores in those with HNPP, the overall burden of HNPP might be higher than previously reported [21]. As with more general neuropathy, disrupted work participation may be an important negative consequence of HNPP [17]. A large portion of participants in the present study with HNPP who reported not working indicated this was because of HNPP (Table 1), though we do not know for how many pain was a factor in this.

Positive correlations were identified between the effect of neurological symptoms on function and pain on function (Table 4). This could be an artifact of the use of a modified version of the BPI-PIS for neurological interference. Taken at face value though, this indicated that the burden of pain across multiple facets of life for people with HNPP is likely 
to equal the impact of any neurological symptoms. Further studies using global measures of health related quality of life would be important.

\section{Strengths and limitations}

Only people with genetically established diagnosis of HNPP were included in this study. Our definition of the pain group as having persistent pain and having experienced pain in the last week is likely to have defined a cohort that excluded more trivial or episodic pain that is frequently less burdensome and potentially not clinically important. We have characterised pain with established classification protocols, providing increased information on potential pain mechanisms in HNPP than previously found in the literature. However, it should be acknowledged that the precise validity and reliability of these questionnaires in HNPP have not been established, though broader applicability has. In addition, the PD-Q and CSI should be considered as screening tool for the identification of underlying pain mechanisms, but not diagnostic tools. Hence these questionnaires do not substitute the clinical diagnosis of neuropathic pain and central sensitisation. Future studies should include a clinical assessment and detailed sensory profiling for the pain characterisation of individuals with HNPP. Further, our measure of interference from neurological symptoms was derived from the BPI-PIS, which, while suitable for the aims of this study, may need expansion in future research.

\section{Conclusion}

In this cohort of people with HNPP, persistent pain was common and had significant negative impact. Pain characterisation identified neuropathic pain and/or central sensitisation as common, potential underlying processes. Pain may plausibly be directly 
related to the underlying pathophysiology of HNPP. Further consideration of including pain as a primary symptom of HNPP is warranted.

\section{Conflict of Interest}

On behalf of all authors, the corresponding author states that there is no conflict of interest.

\section{References}

1. Bird TD (2016) Hereditary Neuropathy with Liability to Pressure Palsies. In: Pagon RA, Adam MP, Ardinger HH, Wallace SE, Amemiya A, Bean LJH, Bird TD, Ledbetter N, Mefford HC, Smith RJH, Stephens K (eds) GeneReviews(R). University of Washington, Seattle, Washington

2. Branco JC (2010) State-of-the-art on fibromyalgia mechanism. Acta Reumatol Port 35:10-15

3. Burns RJ (2000) Introduction To Research Methods. Pearson Education Australia, NSW, Australia

4. Chance PF (2006) Inherited focal, episodic neuropathies: hereditary neuropathy with liability to pressure palsies and hereditary neuralgic amyotrophy. Neuromolecular Med 8:159-174

5. Chance PF (1999) Overview of hereditary neuropathy with liability to pressure palsies. Ann N Y Acad Sci 883:14-21 
6. Choi YH, Dunn B (2016) Low back pain with radicular symptoms as a presentation of hereditary neuropathy with liability to pressure palsies: the diagnostic challenge of an atypical presentation. Muscle Nerve 53:655-656

7. Clauw DJ (2009) Fibromyalgia: an overview. Am J Med 122:S3-S13

8. Cleeland CS (2009) The Brief Pain Inventory: Users Guide. In:The University of Texas, Houston, Texas

9. de Oliveira AP, Pereira RC, Onofre PT, Marques VD, de Andrade GB, Barreira AA, Marques Junior W (2016) Clinical and neurophysiological features of the hereditary neuropathy with liability to pressure palsy due to the $17 \mathrm{p} 11.2$ deletion. Arq Neuropsiquiatr 74:99-105

10. Dubourg O, Mouton P, Brice A, LeGuern E, Bouche P (2000) Guidelines for diagnosis of hereditary neuropathy with liability to pressure palsies. Neuromuscul Disord 10:206-208

11. Freynhagen R, Baron R, Gockel U, Tolle TR (2006) painDETECT: a new screening questionnaire to identify neuropathic components in patients with back pain. Curr Med Res Opin 22:1911-1920

12. Jensen TS, Baron R, Haanpaa M, Kalso E, Loeser JD, Rice AS, Treede RD (2011) A new definition of neuropathic pain. Pain 152:2204-2205 
13. Li J, Krajewski K, Lewis RA, Shy ME (2004) Loss-of-function phenotype of hereditary neuropathy with liability to pressure palsies. Muscle Nerve 29:205210

14. Maier C, Baron R, Tolle TR, Binder A, Birbaumer N, Birklein F, Gierthmuhlen J, Flor H, Geber C, Huge V, Krumova EK, Landwehrmeyer GB, Magerl W, Maihofner C, Richter H, Rolke R, Scherens A, Schwarz A, Sommer C, Tronnier V, Uceyler N, Valet M, Wasner G, Treede RD (2010) Quantitative sensory testing in the German Research Network on Neuropathic Pain (DFNS): somatosensory abnormalities in 1236 patients with different neuropathic pain syndromes. Pain 150:439-450

15. Marchettini P, Lacerenza M, Mauri E, Marangoni C (2006) Painful peripheral neuropathies. Curr Neuropharmacol 4:175-181

16. Mayer TG, Neblett R, Cohen H, Howard KJ, Choi YH, Williams MJ, Perez Y, Gatchel RJ (2012) The development and psychometric validation of the central sensitization inventory. Pain Pract 12:276-285

17. McDermott AM, Toelle TR, Rowbotham DJ, Schaefer CP, Dukes EM (2006) The burden of neuropathic pain: results from a cross-sectional survey. Eur J Pain 10:127-135

18. Meretoja P, Silander K, Kalimo H, Aula P, Meretoja A, Savontaus ML (1997) Epidemiology of hereditary neuropathy with liability to pressure palsies (HNPP) in south western Finland. Neuromuscul Disord 7:529-532 
19. Neblett R, Cohen H, Choi Y, Hartzell MM, Williams M, Mayer TG, Gatchel RJ (2013) The Central Sensitization Inventory (CSI): establishing clinically significant values for identifying central sensitivity syndromes in an outpatient chronic pain sample. J Pain 14:438-445

20. Nijs J, De Kooning M, Beckwee D, Vaes P (2015) The neurophysiology of pain and pain modulation: modern pain neuroscience for musculoskeletal therapists. In: Jull G, Moore A, Falla D, Lewis J, McCarthy C, Sterling M (eds) Grieve's Modern Musculoskeletal Physiotherapy. Elsevier Health Sciences, London, UK, pp 8-18

21. Padua L, Pazzaglia C, Cavallaro T, Commodari I, Pareyson D, Quattrone A, Rizzuto N, Vita G, Tonali PA, Schenone A (2007) Quality of life is not impaired in patients with hereditary neuropathy with liability to pressure palsies. Eur J Neurol 14:e45-e46

22. Radbruch L, Loick G, Kiencke P, Lindena G, Sabatowski R, Grond S, Lehmann KA, Cleeland CS (1999) Validation of the German version of the Brief Pain Inventory. J Pain Symptom Manage 18:180-187

23. Schenone A, Nobbio L, Caponnetto C, Abbruzzese M, Mandich P, Bellone E, Ajmar F, Gherardi G, Windebank AJ, Mancardi G (1997) Correlation between PMP-22 messenger RNA expression and phenotype in hereditary neuropathy with liability to pressure palsies. Ann Neurol 42:866-872

24. Siddall PJ, Cousins MJ (2004) Persistent pain as a disease entity: implications for clinical management. Anesth Analg 99:510-520 
25. Suzuki R, Dickenson A (2005) Spinal and supraspinal contributions to central sensitization in peripheral neuropathy. Neurosignals 14:175-181

26. Takahashi S, Chum M, Kimpinski K (2017) Electrodiagnostic Characterization of Hereditary Neuropathy With Liability to Pressure Palsies. J Clin Neuromuscul Dis $18: 119-124$

27. Tampin B, Bohne T, Callan M, Kvia M, Melsom Myhre A, Neoh EC, Bharat C, Slater H (2017) Reliability of the English version of the painDETECT questionnaire. Curr Med Res Opin 33:741-748

28. Tan G, Jensen MP, Thornby JI, Shanti BF (2004) Validation of the Brief Pain Inventory for chronic nonmalignant pain. J Pain 5:133-137

29. Ueda H, Matsunaga H, Olaposi OI, Nagai J (2013) Lysophosphatidic acid: chemical signature of neuropathic pain. Biochim Biophys Acta 1831:61-73

30. Yilmaz U, Bird TT, Carter GT, Wang LH, Weiss MD (2015) Pain in hereditary neuropathy with liability to pressure palsy: an association with fibromyalgia syndrome? Muscle Nerve 51:385-390

31. Yurrebaso I, Casado OL, Barcena J, Perez de Nanclares G, Aguirre U (2014) Clinical, electrophysiological and magnetic resonance findings in a family with hereditary neuropathy with liability to pressure palsies caused by a novel PMP22 mutation. Neuromuscul Disord 24:56-62 
32. Zelman DC, Dukes E, Brandenburg N, Bostrom A, Gore M (2005) Identification of cut-points for mild, moderate and severe pain due to diabetic peripheral neuropathy. Pain 115:29-36 
Figure 1:

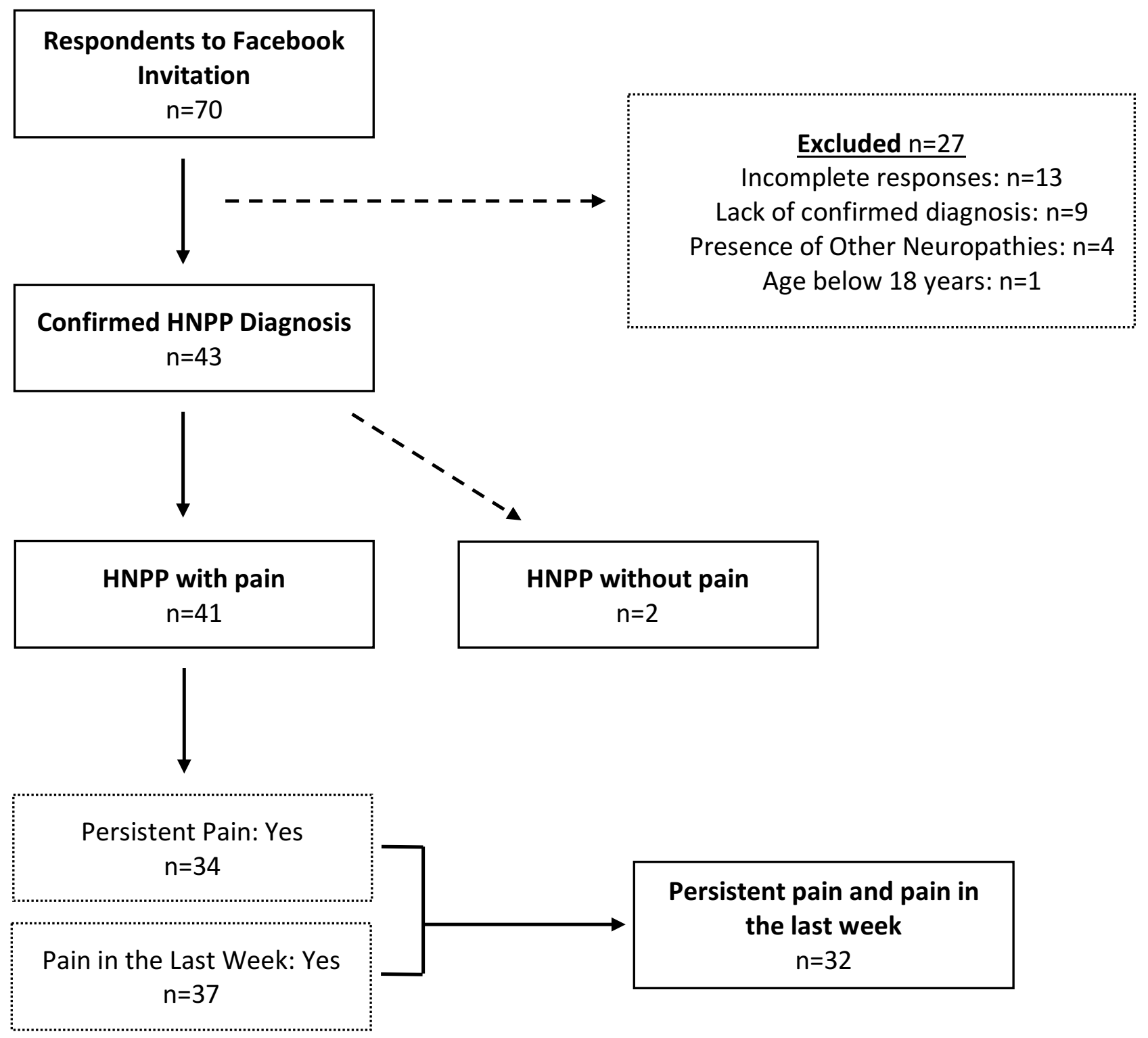


Table 1: Participant characteristics ${ }^{a}$ of the total cohort $(n=43)$ and of participants who reported persistent pain with the experience of pain in the last week $(n=32)$

\begin{tabular}{lcc}
\hline & Total Cohort & $\begin{array}{c}\text { Persistent pain experienced } \\
\text { in the last week }\end{array}$ \\
\hline Age (years) & $47(14)$ & $48(12)$ \\
Females & $34(79 \%)$ & $25(78 \%)$ \\
Time since diagnosis (years) & $9(9)$ & $8(7)$ \\
Not Working & $26(60 \%)$ & $21(66 \%)$ \\
- HNPP the reason for not working & $15(58 \%)$ & $14(67 \%)$ \\
Reported Neurological Symptoms & & \\
- Tingling & $41(95 \%)$ & $32(100 \%)$ \\
- Pins and Needles & $42(98 \%)$ & $32(100 \%)$ \\
- Numbness & $43(100 \%)$ & $32(100 \%)$ \\
- Twitching & $36(84 \%)$ & $28(88 \%)$ \\
- Weakness & $43(100 \%)$ & $32(100 \%)$ \\
Health Professional Diagnosed & & \\
Comorbidity & & $6(19 \%)$ \\
- Heart/Circulatory Disorder & $8(19 \%)$ & $2(6 \%)$ \\
- Diabetes & $3(7 \%)$ & $4(12 \%)$ \\
- Fibromyalgia & $4(9 \%)$ & $10(31 \%)$ \\
- Arthritis & $12(28 \%)$ & $13(41 \%)$ \\
- Irritable Bowel Syndrome & $16(37 \%)$ & $13(41 \%)$ \\
- Headaches/Migraine & $16(37 \%)$ & $18(56 \%)$ \\
- Depression/Anxiety & $23(53 \%)$ & $9(28 \%)$ \\
- Sleep Disorder & $10(23 \%)$ &
\end{tabular}

${ }^{a}$ Reported as mean (standard deviation) or $\mathrm{n}(\%)$ according to data type.

${ }^{b} \mathrm{n}=26$

Table 2: Body regions in which participants experienced neurological symptoms and pain in the last week $(n=32)$.

\begin{tabular}{ccc}
\hline Body Area & $\begin{array}{c}\text { Neurological Symptoms } \\
\mathrm{n}(\%)\end{array}$ & $\begin{array}{c}\text { Pain } \\
\mathrm{n}(\%)\end{array}$ \\
\hline Head or face & $15(47 \%)$ & $6(19 \%)$ \\
Neck & $10(31 \%)$ & $19(59 \%)$ \\
Left shoulder & $10(31 \%)$ & $18(56 \%)$ \\
Right Shoulder & $10(31 \%)$ & $17(53 \%)$ \\
Left arm/hand & $30(94 \%)$ & $22(69 \%)$ \\
Right arm/hand & $28(88 \%)$ & $25(78 \%)$ \\
Upper back & $7(22 \%)$ & $14(44 \%)$ \\
Lower Back & $12(38 \%)$ & $23(72 \%)$ \\
Left leg/foot & $29(91 \%)$ & $28(88 \%)$ \\
Right leg/foot & $30(94 \%)$ & $26(81 \%)$ \\
\hline
\end{tabular}


Table 3: Pain characterization in participants with HNPP experiencing persistent pain in the last week. For comparison, interference related to neurological symptoms is also reported. $\left(n=32^{a}\right)$

\begin{tabular}{lc} 
Brief Pain Inventory & \\
- Pain Severity Score & $4.9(1.9)$ \\
- Pain Interference Score & $5.5(2.5)$ \\
Neurological Interference Score & $5.5(2.2)$ \\
painDETECT Categorisation & \\
- Neuropathic unlikely & $1(3 \%)$ \\
- Unclear & $7(22 \%)$ \\
- Neuropathic likely & $24(75 \%)$ \\
Central Sensitization Inventory Categorisation & \\
- Central sensitisation unlikely & $5(16 \%)$ \\
- Central sensitisation likely & $27(84 \%)$ \\
Pain related to HNPP & \\
- Always & $19(59 \%)$ \\
- Sometimes & $13(41 \%)$ \\
- Never & $0(0 \%)$ \\
Sought medical assistance for pain & \\
- Yes & $28(88 \%)$ \\
- No & $4(12 \%)$ \\
\hline a Reported as mean (standard deviation) or $n$ (\%) according to data type.
\end{tabular}

${ }^{a}$ Reported as mean (standard deviation) or $\mathrm{n}(\%)$ according to data type.

Table 4: Correlation (Pearson's) between neurological symptom interference and pain interference, in participants with HNPP who had persistent pain and pain in the last week. $(n=32)$

\begin{tabular}{llc}
\hline Interference Dimensions & $\mathbf{r}^{\mathbf{2}}$ & $\mathbf{p}$ \\
\hline General activity & .37 & .037 \\
Mood & .77 & .018 \\
Walking ability & .59 & $<.001$ \\
Normal work & .69 & $<.001$ \\
Relations with other people & .74 & $<.001$ \\
Sleep & .77 & $<.001$ \\
Enjoyment of life & .86 & $<.001$ \\
TOTAL SCORE & .73 & $<.001$ \\
\hline
\end{tabular}

